\title{
Equilibrium and dynamics in the transport of ultrarelativistic charged beams
}

\author{
R.P. Nunes, ${ }^{1, *}$ R. Pakter, ${ }^{2}$ F. B. Rizzato, ${ }^{2, \dagger}$ and A. Endler ${ }^{2}$ \\ ${ }^{1}$ Departamento de Engenharia Elétrica, Universidade Federal do Rio Grande do Sul, \\ Avenida Osvaldo Aranha, 103, 90035-190, Porto Alegre, RS, Brasil \\ ${ }^{2}$ Instituto de Física, Universidade Federal do Rio Grande do Sul, P.O. Box 15051, 91501-970, Porto Alegre, RS, Brasil
}

(Received 29 April 2011; published 24 May 2012)

\begin{abstract}
The present work explores the full role of relativistic effects in the transport of magnetically focused non-neutral cold beams. Not only relativistic effects along the transport axis are discussed, but relativistic effects associated with the transverse particle dynamics are also investigated. Transverse effects are directly connected with the amount of charge the beam transports and a proper discussion of relativistic features should include accurate analysis of all fields self-consistently created by space charge. We review and adapt the equilibrium analysis, and proceed to develop a convenient method to tackle dynamical situations. Simulations revealing how flattop initial conditions, the typical equilibrium profiles of nonrelativistic beams, evolve toward highly nonlinear states in relativistic beams are then presented and discussed.
\end{abstract}

DOI: 10.1103/PhysRevSTAB.15.054404

PACS numbers: 41.75.Ht, 29.27.Ac

\section{INTRODUCTION}

The present work investigates the paraxial transport of initially cold relativistic beams along uniform solenoidal magnetic focusing channels. In earlier works on this kind of beam dynamics [1-3], relativistic effects in the transverse dynamics were neglected. Here, we specifically examine such effects in cases where the longitudinal beam velocity is highly relativistic, and charge densities are sufficiently small that radial velocities remain small as compared with the longitudinal velocity. Beams of this kind have small perveances and emittances, or large focusing strengths. Perveance, which we define formally later, is a quantity related to the beam charge, and emittance is a measure of the beam transverse temperature [3].

One should realize that relativistic effects in the transverse plane become relevant if perveance is relatively small, provided the beam is already moving with ultrarelativistic longitudinal speeds along the transport axis. This is the scenario for accelerator systems [1] and devices like free-electron lasers with solenoids for emittance compensation [4], for instance. Here, the added transverse effects induced by the space charge, and quantitatively represented by perveance, may be sufficient to affect the transverse velocity and cause large variations in $\gamma$. The respective perveances are low as a result of the extremely large velocities and fall within the range discussed in the present analysis [5]. In addition, smooth focusing

\footnotetext{
*roger.pizzato@ufrgs.br

†rizzato@if.ufrgs.br
}

Published by the American Physical Society under the terms of the Creative Commons Attribution 3.0 License. Further distribution of this work must maintain attribution to the author(s) and the published article's title, journal citation, and DOI. approximations for the focusing field are appropriate in these cases. Smooth focusing approximates the periodic focusing field by a constant longitudinal magnetic field and is quite compatible with fast beams [3]. In more objective terms, we shall be interested in regimes where emittance is low, focusing is strong, and perveance is sufficient to have pronounced transverse relativistic space-charge effects, yet not too strong to break the paraxial approximation.

We see from all the previous remarks that beams can be simply referred to as either relativistic or nonrelativistic, as far as the transverse dynamics is concerned. We shall adopt this terminology pointing out that the longitudinal velocity can be large even in the nonrelativistic case.

The space-charge fields, which play an important role in relativistic regimes, must satisfy the boundary conditions on the walls of conventional conducting pipes surrounding the beam. Conductors are grounded, so the zero potential condition at $r=r_{w}$ has to be satisfied. $r_{w}$ is the pipe radius in the axisymmetric cylindrical geometries we shall explore here. Conditions on the various components of the magnetic vector potential must also be satisfied when perveances are large enough to give rise to self-consistent effects [6]. In nonrelativistic cases, and depending on the symmetries of system, it may be easier to deal with fields rather than with the potentials [3]. In the present analysis we shall make use of a Hamiltonian formulation where use of potentials is much more convenient. The formalism therefore includes potentials and the respective boundaries, both in equilibrium and dynamical states.

Fully relativistic dynamics has been investigated in the past within the context of beam equilibria [1,7-9]. The present work reviews relativistic beam equilibria from a slightly different perspective and extends the analysis to dynamical states for which we develop the appropriate 
numerical code. Dynamics shall be investigated for ultrarelativistic beams where even small perveances play a noticeable role in the associated transverse relativistic dynamics. Since perveances are small, and while the beam remains space-charge dominated with small emittances, paraxial approximations are accurate to describe transport. Then, one can neglect longitudinal field derivatives as compared to transverse gradients across the beam. However, as mentioned in the preceding paragraph, all self-fields must be correctly evaluated-including diamagnetic corrections to the focusing field-since all depend on the influential effects of perveance.

The Hamiltonian method developed here adds relativistic transverse effects to the well-known nonrelativistic transverse dynamics of paraxial beams. This approach allows for a closer comparison with previous nonrelativistic models of paraxial beams [1,3] where the formalism is similar, as seen in the Hamiltonian formulation discussed in Ref. [3], for instance. Other relativistic models and methods employ techniques not so similar to the ones discussed in the present paper [10-12].

We finally apply the analytical formalism and numerical technique to see how beam equilibrium and beam dynamics changes when relativity is taken into account. First of all, our code is used with conveniently added dissipative forces to reach equilibrium. The dissipative force is implemented by a velocity-dependent term and causes the system to fall into equilibrium. The equilibrium agrees with the analytical prediction and does not display the flattop profile of nonrelativistic approximations [13]. Then, as one turns off dissipation and starts from offequilibrium initial conditions, the conservative dynamics is shown to freely evolve to wave-breaking nonlinear states [14] where the beam develops density singularities along the radial coordinate with subsequent ejection of particles. Wave-breaking states are otherwise present both in nonrelativistic approximations for beams [4,15-17] as well as in one-dimensional relativistic models [18], when some nonuniformity in the initial conditions are considered. Here we show that, as relativistic effects are fully considered, even uniform flattop profiles evolve to wavebreaking conditions and eventual relaxation.

The paper is organized in the following way. In Sec. II we introduce and discuss the model, in Sec. III we review equilibria of relativistic beams, in Sec. IV we develop, test, and apply the numerical method, and in Sec. V we conclude the work.

\section{MODEL}

The appropriate variables describing general steadystate mismatched beams are more easily thought as evolving with the axial coordinate rather than with the time. We shall therefore adopt the modified Hamiltonian formalism where the longitudinal coordinate $z$ along the focusing axis plays the role of "time" [3]. Our basic assumptions to perform the analysis are: (i) small perveances and emittances, (ii) smooth focusing, and (iii) axisymmetry.

The perveance $K$ is defined as basically the ratio of the beam transverse electromagnetic energy to its longitudinal energy: in cgs units, $K \equiv 2 N q^{2} / m c^{2} \beta_{0}^{2} \gamma_{0}^{3} . N$ is the number of particles per unit longitudinal length, $q$ and $m$ are, respectively, the charge and mass of beam particles, and $\beta_{0}$ is the injection velocity normalized to the speed of light $c$. The relativistic factor associated with $\beta_{0}$ is $\gamma_{0}=$ $1 / \sqrt{1-\beta_{0}^{2}}$.

The physical meaning of the perveance allows us to conclude that, for small emittance, when $K \ll 1$ then $|d r / d z| \ll 1$ as well $[1,3]$. The latter inequality forms the basis of the paraxial approximation.

In this modified Hamiltonian formalism, the Hamiltonian is the negative of the longitudinal momentum, $\mathcal{H}=-P_{z}$, which can be promptly written from the original electromagnetic Hamiltonian $H$ under the conditions to be discussed below [3]:

$$
\mathcal{H}=-m c \sqrt{\frac{(H-q \phi)^{2}}{m^{2} c^{4}}-1-\frac{P_{r}^{2}}{m^{2} c^{2}}-\frac{q^{2} A_{\theta}^{2}}{m^{2} c^{4}}}-\frac{q}{c} A_{z}(r) .
$$

$\varphi$ and $\mathbf{A}$ are the self-consistently calculated electrostatic and vector potentials, from which forces can be eventually obtained. Since the beam is assumed to be steady state and axisymmetric, the original Hamiltonian $H$ and the azimuthal canonical angular momentum are both conserved. At beam entrance where focusing fields are absent, we assume injection at the same energy level $H$ (written for future purposes as $H=m c^{2} \gamma_{0}$ ) and zero angular momentum for all particles, from which follows $P_{\theta}=0$ and the final expression (1) for $\mathcal{H}$. The Coulomb gauge is chosen and $A_{r}$ is neglected in the paraxial approximation. We also recall that $P_{\theta}$ is the canonical azimuthal angular momentum, which is written in terms of the associated kinetic momentum $p_{\theta}$ as $P_{\theta}=r p_{\theta}+(q / c) r A_{\theta}$. $A_{\theta}$ includes an external contribution $A_{\theta \text {,external }}=r B_{0} / 2$ generating the applied focusing magnetic field $\mathbf{B}_{0}=B_{0} \hat{\mathbf{z}}$, and all relevant diamagnetic corrections generated by the self-field contributions to $A_{\theta}$.

One sees that the remaining canonical coordinates of the formalism are the radial coordinate $r$ and the conjugate momentum $P_{r}$, whose evolution can be obtained from the canonical expressions,

$$
\begin{gathered}
r^{\prime}=\frac{\partial \mathcal{H}}{\partial P_{r}}=\frac{P_{r}}{p_{z}} \\
P_{r}^{\prime}=-\frac{\partial \mathcal{H}}{\partial r}=-\frac{q^{2}}{c^{2}} \frac{A_{\theta}}{p_{z}} \frac{\partial A_{\theta}}{\partial r}-\frac{q}{v_{z}} \frac{\partial \phi}{\partial r}+\frac{q}{c} \frac{\partial A_{z}}{\partial r} .
\end{gathered}
$$


In expressions (2) and (3), primes denote derivatives with respect to $z, p_{z}=-\mathcal{H}-q / c A_{z}$ is the longitudinal kinetic momentum, and the longitudinal velocity is simply obtained from the additional canonical equation,

$$
1 / v_{z}=t^{\prime}=-\partial \mathcal{H} / \partial H=(H-q \varphi) / c^{2} p_{z} .
$$

The relevant field equations are obtained from

$$
\begin{aligned}
& \frac{1}{r} \frac{\partial}{\partial r} r \frac{\partial \varphi}{\partial r}=-4 \pi q n, \\
& \frac{1}{r} \frac{\partial}{\partial r} r \frac{\partial A_{z}}{\partial r}=-\frac{4 \pi}{c} j_{z} \\
& \frac{\partial}{\partial r} \frac{1}{r} \frac{\partial r A_{\theta}}{\partial r}=-\frac{4 \pi}{c} j_{\theta} .
\end{aligned}
$$

$n=n(r, z)$ is the radially dependent density, and $\mathbf{j}=q \mathbf{v} n$ is the current density with $\mathbf{v}$ as the average particle velocity.

\section{REVIEW OF EQUILIBRIUM STATES}

Equilibrium states for ultrarelativistic beams have been obtained earlier with a formulation more directly based on the relevant components of the electric and magnetic fields [1].

Our dynamical equations to be analyzed in the following sections are based on a Hamiltonian formulation and the respective field potentials. Therefore we devote this section to reformulate the equilibrium theory in terms of the appropriate electric and magnetic potentials.

For equilibrium states, where $z$ dependence and $A_{r}$ are truly absent, the set of equations (5)-(7) is exact and can be reduced along the following lines. We start by making use of a cold beam approach, where there is no velocity dispersion for particles at each radial coordinate.

Then we first observe that, since an equilibrium condition is being sought, the pair of equations (2) and (3) informs that $P_{r} \rightarrow 0$ and that $\partial \mathcal{H} / \partial r=0$, i.e., $\mathcal{H}$ is a constant independent of the radial coordinate. We next recall that the potential $\varphi$ vanishes on the conducting wall at $r_{w}: \varphi\left(r=r_{w}\right)=0$. One also realizes that since $A_{z}$ enters the theory in the form of an added function in the Hamiltonian (1), and in the form of a derivative in Eq. (6), it is defined up to a constant term which we take to be such that $A_{z}\left(r=r_{w}\right)=0$.

If we combine Eqs. (5) and (6) using the expressions for the velocity $v_{z}$ and $\mathcal{H}$, one arrives at the equality

$$
\frac{q}{c} \frac{\partial}{\partial r} r \frac{\partial A_{z}}{\partial r}=-q\left(\frac{\mathcal{H}+\frac{q}{c} A_{z}}{H-q \varphi}\right) \frac{\partial}{\partial r} r \frac{\partial \varphi}{\partial r} .
$$

Using standard properties involving derivatives of products of functions, and recalling that $H$ and $\mathcal{H}$ are constants, Eq. (8) can be solved as

$$
\frac{\mathcal{H}+\frac{q}{c} A_{z}}{H-q \varphi}=\frac{\mathcal{H}}{H} \Rightarrow A_{z}=\beta_{e q} \varphi
$$

when the boundary conditions are implemented. We note that $\beta_{\mathrm{eq}}$ is the value of $\beta_{z}$ in equilibrium conditions. Equation (9) then allows one to express $A_{z}$ in terms of $\varphi$, with $\beta_{\text {eq }} \equiv-c \mathcal{H} / H$ as the common longitudinal velocity of all beam particles. Therefore, regardless of the density profile across the beam, we find that the longitudinal speed at equilibrium follows a uniform distribution as a function of the radial coordinate [1,7].

Since it is possible to express $A_{z}$ in terms of $\varphi$, convenient handling of the constant Hamiltonian $\mathcal{H}$ defined by expression (1) allows one to write the scalar potential $\varphi$ entirely in terms of the azimuthal component of the vector potential, $\varphi(r)=\varphi\left[A_{\theta}(r)\right]$.

The next step in our procedure is once again use Eq. (5), now in Eq. (7), recalling that owing to the conserved azimuthal canonical momentum, $v_{\theta}=-q / m c\left(A_{\theta} / \gamma\right)$. This leads directly to a self-contained differential equation for $A_{\theta}$,

$$
\frac{\partial^{2} A_{\theta}}{\partial r^{2}}+\frac{1}{r} \frac{\partial A_{\theta}}{\partial r}+\frac{A_{\theta}}{r^{2}}=\frac{4 \pi q^{2} n\left(A_{\theta}, \frac{\partial A_{\theta}}{\partial r}, \frac{\partial^{2} A_{\theta}}{\partial r^{2}}\right) A_{\theta}}{H-q \varphi\left(A_{\theta}\right)},
$$

where the density $n(r)=n\left(A_{\theta}, \partial A_{\theta} / \partial r, \partial^{2} A_{\theta} / \partial r^{2}\right)$ is promptly obtained, as one combines Eqs. (1) and (5).

Equation (10) is complicated, but since one knows how to express $\varphi$ and $n$ in terms of $A_{\theta}$ and its radial derivatives through Eqs. (1) and (5), it can be solved once proper boundary conditions are imposed.

As mentioned earlier, the beam extends itself up to a radius $r=r_{b}$ yet unknown, and at the conductor wall the electric potential $\varphi$ vanishes. Gauss's law applied to the cylindrical geometry therefore reveals that $r_{b} \partial \varphi\left(r_{b}\right) / \partial r_{b}=-2 N q$, where we recall that $N$ is the number of particles per unit longitudinal length. The condition on the wall potential requires that $\varphi\left(r_{w}\right)=0$ so one concludes that in the vacuum region $\varphi\left(r_{b}<r<r_{w}\right)=$ $-2 N q \log \left(r / r_{w}\right)$. In addition, since the azimuthal vector potential $A_{\theta}$ now becomes an active variable in the problem, its boundary conditions must be examined too. At the origin the line integral of $A_{\theta}$ encircles a magnetic flux that grows with the cross sectional area $\sim r^{2}$. One thus concludes that $A_{\theta}(r=0)=0$ [with $A_{\theta}(r \rightarrow 0) \sim r$ ]. At the beam border $r=r_{b}$ the magnetic field must attain its given vacuum value $B_{0}$. Note that the beam modifies the longitudinal component of the applied field only in its inner region. This can be seen as one realizes that, as far as the azimuthal dynamics is considered, the beam is similar to a collection of current carrying solenoids with resulting zero longitudinal field in the outer region. Equivalently, the selffield contribution in Eq. (10) varies as 1/ $r$ outside the beam where the source density is null, which causes the respective magnetic field to vanish. The solenoidal magnetic 
field, on the other hand, is generated both in the inner and outer regions of the beam.

Summing up our reasoning, for a given injection energy we wish to find out the values of three adjustable unknowns that allow for the simultaneous fulfillment of the three conditions below (in addition to the fixed condition determining $A_{\theta}$ at the origin):

$$
\begin{gathered}
r_{b} \frac{\partial \varphi\left(r_{b}\right)}{\partial r_{b}}=-2 N q, \\
\varphi\left(r_{b}\right)=-2 N q \log \left(r_{b} / r_{w}\right), \\
\frac{A_{\theta}\left(r_{b}\right)}{r_{b}}+\frac{\partial A_{\theta}\left(r_{b}\right)}{\partial r_{b}}=B_{0} .
\end{gathered}
$$

For the numerical survey we will conduct shortly, we fix values for $N$ and $r_{w}$ and solve Eq. (10) at an arbitrary radial coordinate, and for arbitrary parameters $\beta_{\mathrm{eq}}$ and $\partial A_{\theta}(0) / \partial r$, the latter related to the magnetic field on the axis. Then we finally set up a root finder scheme for these parameters $\beta_{\text {eq }}$ and $\partial A_{\theta}(0) / \partial r$ along with the beam radius $r_{b}$, based on conditions (11)-(13). The root finder scheme is implemented with algebraic mathematical softwares like MATHEMATICA. We first solve for $A_{\theta}$ and $\varphi$ in terms of the unknown variables and finally obtain their numerical values from Eqs. (11)-(13). Initial guesses are provided by the nonrelativistic approximation. Once again, we point out that the radial derivative of $A_{\theta}$, one of the variables to be determined, represents the modified focusing field at beam axis.

In the following we examine dynamical states of relativistic beams.

\section{NUMERICAL METHODS FOR DYNAMICS}

Dynamics has been well investigated under the nonrelativistic approximation, which amounts to taking only first-order linear terms in $\mathcal{H}$ to describe the transverse motion $[1,3]$. This procedure is appropriate to describe short time scale features of beams with moderately large longitudinal speeds, where the dominant terms in $\gamma$ are the linear ones. However, if one thinks, for instance, of a situation where the beam is injected at the equilibrium condition of the linear approximation, which is an inexact equilibrium for the full problem, one relevant question would be on how the exact system evolves since in this case dynamics is driven solely by relativistic effects.

To address the issue, we simulate the full set of equations (2)-(7). Small perveances are assumed, which implies that the paraxial approximation $|d r / d z| \ll 1$ is valid, as long as emittance is also small enough so as not to push initially paraxial particles into nonparaxial orbits. However, corrections to the relativistic factor $\gamma$ are important when ultrarelativistic longitudinal velocities are considered, and are thus kept here.
We take advantage of the cylindrical symmetry, and instead of working with point particles, we use cylindrical particles.

One thousand to 50000 particles are used, depending on the case studied (if laminar equilibrium or wave breaking, respectively). The number of particles is chosen in order to achieve convergence for the simulations. Each layer carries a fraction $1 / N_{l}$ of the total charge, with $N_{l}$ denoting the total number of particles. The scalar potential $\varphi$ can be therefore obtained with the help of the corresponding radial Green's function from

$$
\frac{1}{r} \frac{\partial}{\partial r} r \frac{\partial G_{\varphi}\left(r, r^{\prime}\right)}{\partial r}=-4 \pi \frac{1}{r} \delta\left(r-r^{\prime}\right)
$$

with a similar expression for the Green's function $G_{A_{\theta}}$ :

$$
\frac{\partial}{\partial r} \frac{1}{r} \frac{\partial}{\partial r} r G_{A_{\theta}}\left(r, r^{\prime}\right)=-\frac{4 \pi}{c} \frac{1}{r} \delta\left(r-r^{\prime}\right) .
$$

Note that, in contrast to the equilibrium case of Eq. (10), where analysis was performed with a cold-fluid approach, here we make use of Green's function to examine discrete and kinetic effects.

We require that $G_{\varphi}$ is finite for $r<r^{\prime}$ and goes to zero as $r \rightarrow r_{w}$, and that, mimicking the equilibrium state, $G_{A_{\theta}}$ is finite both for $r<r^{\prime}$ and any $r>r^{\prime}$. Welcome simplification arises from the fact that we do not need the full expression for $A_{z}$. Only its radial derivative is needed in the dynamical equations. This derivative is the azimuthal magnetic field, which can be directly calculated with the help of Ampère's law. A word of caution is in order, however. While $\varphi$ can be evaluated from a simple summation over source coordinates involving the corresponding Green's functions $G_{\varphi}$ 's,

$$
\varphi(r)=\frac{N q}{2 \pi N_{l}} \sum_{r^{\prime}} G_{\varphi}\left(r, r^{\prime}\right)
$$

the procedure is far more complicated with the evaluation of $A_{\theta}$. The fact is that, since the source of the original equation (7) involves particle velocities which are proportional to $A_{\theta}$ itself as a result of momentum conservation, calculation must be fully self-consistent:

$$
A_{\theta}(r)=A_{\theta, \text { external }}+\frac{N q^{2}}{2 \pi m c^{2} N_{l}} \sum_{r^{\prime}} G_{A_{\theta}}\left(r, r^{\prime}\right) \frac{A_{\theta}\left(r^{\prime}\right)}{\gamma}
$$

Our strategy to solve the self-consistent equation, Eq. (17), is to iterate the solution at the various positions of the particles, taking the external field as a first approximation. The assumed smallness of the perveance parameter guarantees that the iterative procedure is fast and accurate. In practice only a small number of steps, around five or six, are required. 


\section{A. Recovering equilibrium states}

We now proceed to discuss the numerical analysis involving dynamical simulations. The first step is to compare results of full simulations with the previous survey on equilibrium states. The idea is to provide an extra degree of confidence to our procedures. In order to make the comparisons, we add a dissipative term of the form $-0.1 \kappa P_{r}$ to Eq. (3). The focusing strength in cgs units $\kappa \equiv$ $q^{2} B_{0}^{2} / 4 m^{2} c^{4} \beta_{0}^{2} \gamma_{0}^{2}$ is defined in the context of the nonrelativistic approximation [19,20]. Dissipation included this way has a similar role as in problems of statistical mechanics, pulling the system towards the corresponding equilibrium which can then be compared with the analytical formalism. As an initial condition we choose the cold uniform equilibrium of the nonrelativistic approximation.

The comparison is made in Fig. 1 for $K=0.003$ and $r_{w} / r_{n r}=2$, and shows a remarkable agreement between results based on Eqs. (10)-(13) (thicker line) and simulations (thinner, darker line). Because of the logarithmic dependence of the potential on the wall radius, the analysis is not very sensitive on the ratio $r_{w} / r_{n r}$. The matched beam radius under the nonrelativistic (" $n r$ ") approximation is given by $r_{n r} \equiv \sqrt{K / \kappa}$, and can be used to obtain the associated density in the form $n_{n r}=N / \pi r_{n r}^{2}$. Panel (a) depicts the profile of the full longitudinal focusing magnetic field, we call it $B$, and panel (b) the density $n$. We note that, even for small values of the perveance, accentuated diamagnetic depletion of the focusing magnetic field is already present for the larger $\beta_{0}$ value, $\beta_{0}=0.995$. Also, since focusing is weakened at small values of the radial coordinate, particles attain equilibrium only at larger radii; hence, the noticeable increase of particle densities near the beam border. For the smaller $\beta_{0}$ value, $\beta_{0}=0.95$, one still observes diamagnetic depletion and density nonuniformity. It should be apparent, however, that holding the perveance unchanged, smaller magnitudes of velocity $\beta_{0}$ correspond to gradually more uniform equilibria. In the interest of comparing our results with previous analytical studies for equilibrium states, in panel (c) we superpose the $\beta_{z}=0.995$ curves of Fig. 1(a) with results (filled circles) based on the expression for the magnetic field of Ref. [8]. Panel (c) indicates total agreement.

In the numerical analysis, the focusing factor is absorbed into dimensionless forms of $z, r$, and the full longitudinal magnetic field, as we scale these quantities with respect to $1 / \sqrt{\kappa}, r_{n r}$, and the externally applied longitudinal field $B_{0}$, respectively. Hence, although $\kappa$ is critical to determine the dimensional radius, it is not explicitly present in the dimensionless treatment given to the simulations in the paraxial approximation. The dimensionless quantities $K$ and $\beta_{0}$ are explicitly present in the simulations.

\section{B. Freely evolving beams}

We then remove the dissipation factor and let the system freely evolve from the same homogeneous initial condition (a)

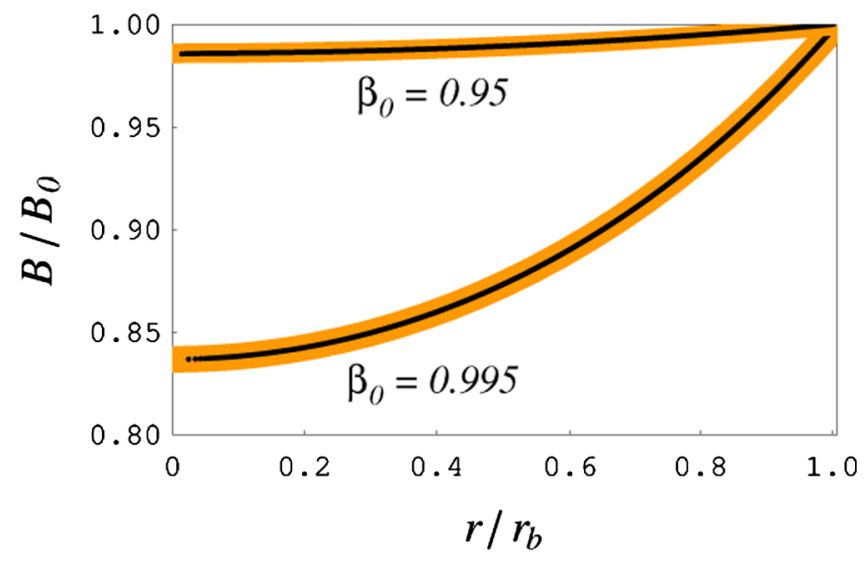

(b)

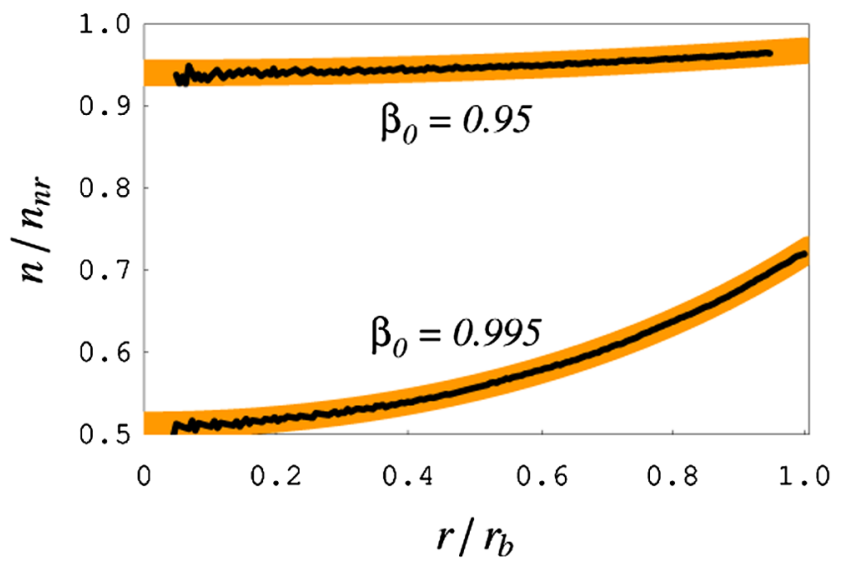

(c)

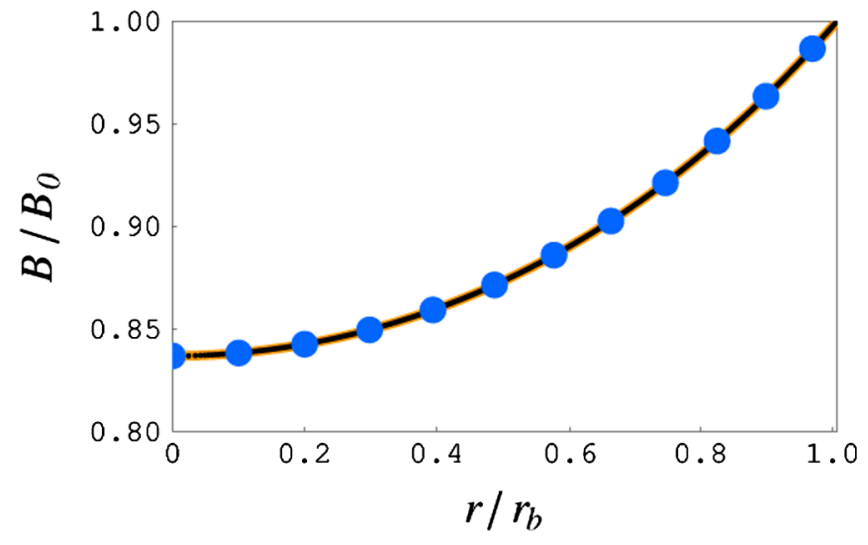

FIG. 1. Equilibrium in ultrarelativistic beams with $K=0.003$. Panel (a) depicts the diamagnetic depletion of the focusing field $B$ while panel (b) plots densities and reveals that particles migrate to higher radii. Relativistic effects are enhanced as velocities increase. In panel (c) we compare the curves of case $\beta_{0}=0.995$ of panel (a) with the magnetic field calculated according to Ref. [8] (filled circles) for the same parameters. Beam terminus at $r_{b} / r_{n r}=1.27\left(\beta_{0}=0.995\right)$ and $r_{b} / r_{n r}=$ $1.024\left(\beta_{0}=0.95\right)$, common values determined both from simulations (thin lines) and analytics (thick lines). In panel (c) thinner lines are employed for better comparison. 
analyzed in the previous subsection with $\beta_{0}=0.995$. The various relativistic nonlinearities of the system introduce several sources of inhomogeneity into the differential equations (2) and (3). We expect the results to be equivalent to those in earlier work [16], where beams ares analyzed using the linear, or nonrelativistic, approach but launched with inhomogeneous initial profiles. Results are displayed in Fig. 2 in the form of a short sequence of phase plots. One indeed notices that the initial flattop profile does not hold still, as density waves are seen to be formed in panel (a) with the eventual creation of a wave-breaking singularity in panel (b) [14]. Particles are ejected, emittance grows from its vanishing initial value in cold beams [16], and the beam ultimately reaches a final relaxed state seen in panel (c) just like the alluded case of beams with inhomogeneous initial profiles. Panel (d), a histogram for the radial distribution of particles, reveals that the salient high-density relaxed core seen in panel (c) follows the same density curve analytically calculated in Fig. 1(b).

This result suggests that a core-halo model with resonant island techniques can be employed to obtain accurate results on the size of the phase space and halo density [21-23]. For convenience in the numerical manipulations, in panel (d) we obtain the density directly from $r^{2}$ histograms of the simulations. Also, in contrast with Fig. 1, radial coordinates are normalized to $r_{n r}$ since we do not have a sharply defined beam radius in the relaxed state.

We thus see that wave breaking in the absence of initial transverse inhomogeneities is possible in relativistic systems. The cause is precisely the relatively strong dependence of the relativistic $\gamma$ factor on the particle velocities, which are themselves affected by the fields.

Even if one starts off with a uniform distribution, nonlinear terms cause the beam to oscillate with nonuniform local frequencies, a process leading to density pileup and

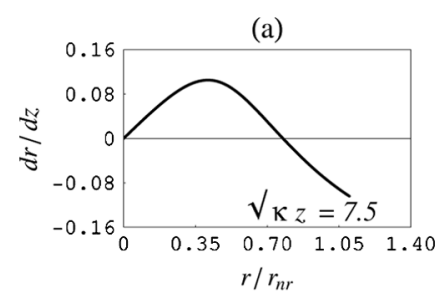

(c)
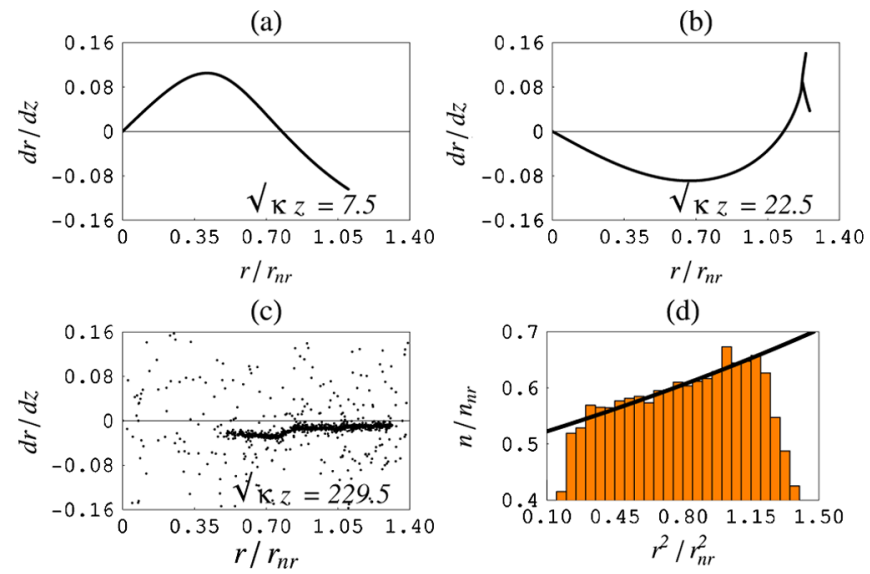

$r / r_{n}$

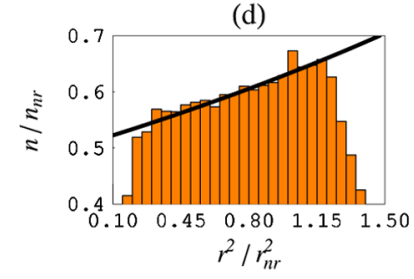

FIG. 2. Various snapshots of the phase space in (a)-(c), including wave breaking in (c). In (d), the density of the relaxed state is compared with the analytical result previously obtained. Here, $\beta_{0}=0.995$ and the other parameters are those used in Fig. 1. concomitant wave breaking, as seen in one-dimensional models with initial small spatial nonuniformities due to mass correction terms [18]. Note that density peaks appear along the radial coordinate but the beam remains smooth and paraxial along the longitudinal axis. Recalling that the equilibrium profile of faster beams becomes increasingly more nonuniform, we expect that, for our initial conditions, wave breaking occurs sooner for faster beams. This conjecture shall be investigated in future publication.

We also note that after relaxation, as in panel (c), some particles move to orbits with larger values of $|d z / d r|$. This suggests a limit to the present theory: if $K$ is so large that a large percentage of particles become nonparaxial with $|d r / d z|>1$, the theory breaks down. This is not the case of the values of $K$ studied in this paper. Here the theory lies within its limits of validity as revealed by the small values of $|d r / d z|$. Smaller values $K$ generate even smaller values of $|d r / d z|$. For small emittances, paraxial behavior can be roughly estimated as we compare an approximation for the magnitude of beam radius, $r_{n r}$, with the longitudinal wavelength $\lambda_{z} \equiv 1 / \sqrt{\kappa}: r_{n r} / \lambda_{z}=\sqrt{\kappa} r_{n r}=\sqrt{K}$. This indicates that the perveance must satisfy $K \ll 1$ for the paraxial approximation [14].

In general, emittance, as measured with our dimensionless variables, relates to the conventional unnormalized emittance [3] through $\varepsilon_{a d}=\varepsilon_{u n} \kappa^{1 / 2} / K$. For mismatches in initially cold beams, emittance is generated by relaxation. For small mismatches, $\varepsilon_{a d}$ is a small dimensionless number and the effects of emittance can be neglected if compared with those associated with the perveance. This case is covered by the preceding estimates. For larger mismatches in initially cold beams, $\boldsymbol{\varepsilon}_{a d}$ becomes larger, but limited by the initial transverse energy of space-charge fields. Therefore, if $K \ll 1$ paraxial approximation applies. For beams initially dominated by emittance effects, $\varepsilon_{a d} \gg 1$, which may be the case of fast beams where $\kappa^{1 / 2}$ goes to zero with $\gamma_{0}$ more slowly than $K ; \kappa^{1 / 2} \sim 1 / \gamma_{0}$ and $K \sim 1 / \gamma_{0}^{3}$. Here, small fluctuation analysis of the beam envelope equation around equilibrium reveals that $d r / d z \sim\left(\varepsilon_{u n} \kappa^{1 / 2}\right)^{1 / 2}=\left(\varepsilon_{a d} K\right)^{1 / 2}$. Paraxial approximation then requires $\varepsilon_{u n} \kappa^{1 / 2} \ll 1$ which can be satisfied for fast beams since $\kappa$ decreases with beam speed. For different reasons, it is necessary, but not sufficient, that $K$ be small also in this case.

Precise analysis of the validity limits demands more numerical work, but initial runs indicate that, in our case, for $K \sim 0.05$ the highest velocity particles in the halo already attain $|d r / d z| \sim 1$. The focus in this work is not properly emittance calculations, and we recall that for the cold beams with small values of $K$ used in this work, the paraxial approximation used in the present investigation is valid. One way to estimate emittance is the core-halo model mentioned earlier.

Our final numerical investigation attempts to provide a numerical reliable basis for our approximations. In Fig. 3 


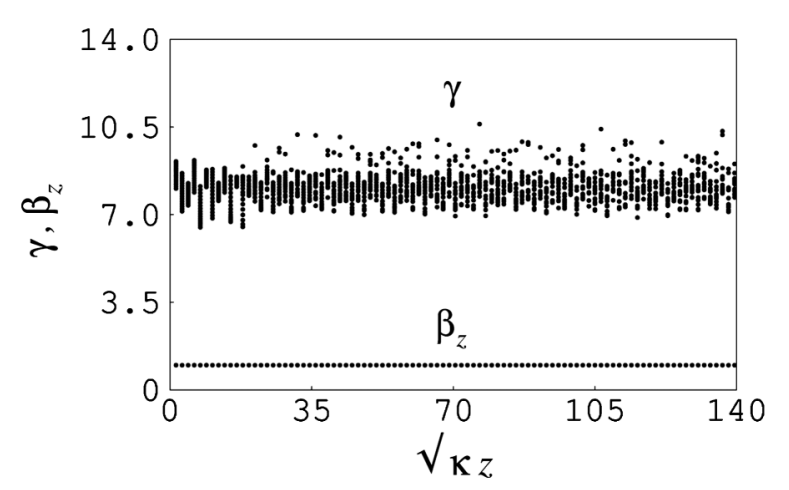

FIG. 3. The time history of $\beta_{z}$ and $\gamma$ for the parameters of Fig. 2.

we plot $\gamma$ and $\beta_{z}$ for all particles as a function of time $\sqrt{\kappa} z$. One sees that although all particles advance with nearly the same and constant velocity along the longitudinal axis, a signature of paraxial behavior, the relativistic $\gamma$ factor fluctuates with noticeable amplitude. Detailed analysis indeed shows that while $\delta \gamma / \gamma \sim 30 \%, \delta \beta_{z} / \beta_{z} \sim 0.1 \%$. Large energy-momentum spreads with simultaneous small velocity spreads are indeed present in known cases of ultrarelativistic beams. These large spreads affect hydrodynamical instabilities of ultrarelativistic beams [24] and are also a feature of electron beams generated in laserplasma accelerators [25]. In any case, we fully confirm our initial remarks where we commented that, while the paraxial approximation is accurate even for ultrarelativistic cases as far as perveances are small, $\gamma$ responds more intensely to all relevant fields. The conclusion is that all velocities, even the longitudinal, must be computed individually since their effect on the respective $\gamma$ 's can largely differ from one particle to another. The present work thus extends previous results where self-consistency and the individual character of particle velocities were not fully considered [26].

\section{FINAL REMARKS}

Non-neutral ultrarelativistic beams are of current interest for accelerator physics [1] and associated relativistic devices like free-electron lasers [27,28], for instance, and in the present paper we investigated the role of relativistic effects in the transverse dynamics of non-neutral beams.

Although only longitudinal relativistic effects are usually considered to be relevant in beams with small perveances, we showed here that even low perveance beams, for which paraxial approximations are accurate, may exhibit noticeable relativistic effects due to the transverse particle dynamics. The key factor is the magnitude of the longitudinal velocity. Transverse relativistic effects will become more prominent as the longitudinal velocity moves up to ultrarelativistic magnitudes. We saw that under these circumstance the focusing field undergoes visible depletion due to diamagnetic effects $[1,8]$, that wave breaking is present even if one starts with the uniform beam equilibrium of nonrelativistic approximations, and that the density profile of both equilibrium and relaxed states diverts from the usual flattop distribution of nonrelativistic regimes. In the opposite case of relatively lower longitudinal velocities, flattop profiles are recovered and diamagnetic depletion drops to much smaller levels. We note that paraxial approximations are accurate up to the beam ends where longitudinal effects become important.

As discussed in Sec. IV the theory has its limitations. $K$ must be small enough that a large majority of particles remains paraxial. The cases studied in this paper satisfy this condition, but as $K$ increases, the number of nonparaxial particles increases as well.

Another limitation is that the present formalism neglects azimuthal inhomogeneities and possible related instabilities. This sort of instabilities is actually present either in completely hollow or filled beams [29,30] and must be examined with care. In the case of filled beams [30], however, azimuthal instabilities appear only for a large value of envelope mismatches.

Similarly to nonrelativistic cases, relaxed states were shown to be a combination of a cold core and hot halo, the cold core resembling the respective equilibrium profile. This suggests that test particle methods can be used to describe the relaxed beam and emittance growth to a high degree of accuracy.

\section{ACKNOWLEDGMENTS}

This work was supported by CNPq and FAPERGS, Brazil, and by the U.S.-AFOSR Grant No. FA9550-09-10283. We wish to thank Yan Levin for a critical reading of the manuscript.

[1] M. Reiser, Theory and Design of Charged Particle Beams (John Wiley, New York, 1994).

[2] C. Chen and R.C. Davidson, Phys. Rev. E 49, 5679 (1994).

[3] R.C. Davidson and H. Qin, Physics of Intense Charged Particle Beams in High Energy Accelerators (World Scientific, Singapore, 2001).

[4] S. G. Anderson and J. B. Rosenzweig, Phys. Rev. ST Accel. Beams 3, 094201 (2000).

[5] S. Humphries, Jr., Nucl. Instrum. Methods Phys. Res., Sect. A 258, 548 (1987).

[6] M. Hess and C. S. Park, IEEE Trans. Plasma Sci. 38, 1584 (2010).

[7] D. C. DePackh and P. B. Ulrich, J. Electron. Control 10, 139 (1961).

[8] P. Diament, Phys. Rev. Lett. 37, 168 (1976).

[9] M. Reiser, Phys. Fluids 20, 477 (1977).

[10] K. V. Lotov, Phys. Plasmas 5, 785 (1998).

[11] J.-L. Vay, Phys. Plasmas 15, 056701 (2008).

[12] G. Lapenta and S. Markidis, Phys. Plasmas 18, 072101 (2011). 
[13] R. P. Nunes, R. Pakter, and F. B. Rizzato, Phys. Plasmas 14, 023104 (2007).

[14] J. M. Dawson, Phys. Rev. 113, 383 (1959).

[15] O. A. Anderson, Part. Accel. 21, 197 (1987).

[16] F. B. Rizzato, R. Pakter, and Y. Levin, Phys. Plasmas 14, 110701 (2007).

[17] E. G. Souza, A. Endler, R. Pakter, F. B. Rizzato, and R. P. Nunes, Appl. Phys. Lett. 96, 141503 (2010).

[18] S. Sengupta, V. Saxena, P. K. Kaw, A. Sen, and A. Das, Phys. Rev. E 79, 026404 (2009).

[19] C. Chen and R. C. Davidson, Phys. Rev. Lett. 72, 2195 (1994).

[20] R. Pakter and F. B. Rizzato, Phys. Rev. E 65, 056503 (2002).

[21] Y. Levin, R. Pakter, and T. N. Teles, Phys. Rev. Lett. 100, 040604 (2008).
[22] R. L. Gluckstern, Phys. Rev. Lett. 73, 1247 (1994).

[23] R. Pakter, G. Corso, T. S. Caetano, D. Dillenburg, and F. B. Rizzato, Phys. Plasmas 1, 4099 (1994).

[24] A. B. Mikhailovskii, Plasma Phys. 23, 413 (1981).

[25] G. Fubiani, J. Qiang, E. Esarey, W. P. Leemans, and G. Dugan, Phys. Rev. ST Accel. Beams 9, 064402 (2006).

[26] R. P. Nunes and F. B. Rizzato, Appl. Phys. Lett. 98, 051501 (2011).

[27] A. Serbeto, J. T. Mendonça, K. H. Tsui, and R. Bonifacio, Phys. Plasmas 15, 013110 (2008).

[28] P. Shukla and B. Eliasson, Phys. Usp. 53, 51 (2010).

[29] J.R. Pierce, IRE Trans. Electron Devices 3, 183 (1956).

[30] W. Simeoni, Jr., F. B. Rizzato, and R. Pakter, Phys. Plasmas 13, 063104 (2006). 